\title{
MicroRNA expression profiling in placenta and maternal plasma in early pregnancy loss
}

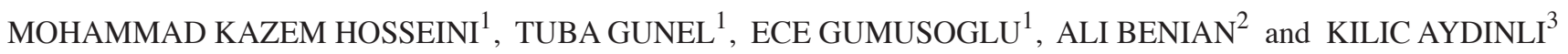 \\ ${ }^{1}$ Department of Molecular Biology and Genetics, Faculty of Science, Istanbul University, \\ Istanbul 34134; ${ }^{2}$ Department of Obstetrics and Gynecology, Cerrahpasa Medical Faculty, \\ Istanbul University, Istanbul 34098; ${ }^{3}$ Medicus Health Center, Istanbul 34365, Turkey
}

Received September 7, 2017; Accepted November 14, 2017

DOI: $10.3892 / \mathrm{mmr} .2018 .8530$

\begin{abstract}
Early pregnancy loss (EPL), also termed early miscarriage, is determined as the unintentional expulsion of an embryo or fetus prior to the 12th week of gestation. EPL frequency is $\sim 15 \%$ in pregnancies. Fetal development and growth is associate with placental function and vessel development; therefore, the placental genome would represent a useful miscarriage model for (epi)genetic and genomic studies. An important factor of placental development and function is epigenetic regulation of gene expression. microRNAs (miRNAs) are the primary epigenetic regulators which have an important role in placental development and function. In the present study, maternal plasma and villous tissue were collected from 16 EPL cases in 6th-8th gestational weeks (GWs) and 8 abortions (control group) in 6th-8th GWs. Detection of the differences in miRNA expression was performed using microarrays and dysregulated miRNAs were validated by reverse transcription-quantitative polymerase chain reaction (RT-qPCR). miRNA microarray findings revealed that four miRNAs, including hsa-miRNA (miR)-125a-3p, hsa-miR-3663-3p, hsa-miR-423-5p and hsa-miR-575 were upregulated in tissue samples. In maternal plasma, two miRNAs (hsa-let-7c, hsa-miR-122) were upregulated and one miRNA (hsa-miR-135a) was downregulated. A total of 6 out of 7 dysregulated miRNAs were validated using RT-qPCR. The target genes of these dysregulated miRNAs were detected using the GeneSpring database. The aim of the present study was to detect dysregulated miRNAs
\end{abstract}

Correspondence to: Dr Mohammad Kazem Hosseini, Department of Molecular Biology and Genetics, Faculty of Science, Istanbul University, Sehzadebası St. Vezneciler, Istanbul 34134, Turkey E-mail: mohammad.hosseini@ogr.iu.edu.tr

Abbreviations: FC, fold-change; miRNAs, microRNAs; MOBG, molecular biology and genetics; NIPD, non-invasive prenatal diagnosis; URSA, unexplained recurrent spontaneous abortion

Key words: early pregnancy loss, microRNA, epigenetics, microarray, maternal plasma, villous tissue in maternal plasma and villous cells and identify the target genes of dysregulated miRNAs and their associated pathways. The target gene analyses have revealed that the affected genes are primarily associated with cell migration, proliferation, implantation, adhesion, angiogenesis and differentiation and all are involved with EPL pathogenesis. Therefore, the present study may contribute to the understanding of the molecular mechanisms which lead to EPL.

\section{Introduction}

Miscarriage or missed abortion is the spontaneous loss of a pregnancy between conception and 20 weeks into the pregnancy, which affects between $\sim 15-20 \%$ women of childbearing age $(1,2)$. The miscarriage until the 12 th week of the pregnancy is termed early pregnancy loss (EPL) which occurs in $~ 15 \%$ of the cases $(3,4)$, and the ones which occur between 12 th and 20 th weeks of the pregnancy are termed late pregnancy loss (LPL) which has an incidence rate of $1-5 \%(5,6)$. It has been previously estimated $\sim 80 \%$ of miscarriages take place in the first 12 weeks (the first trimester) of pregnancy as EPL. The etiological factors during a miscarriage are generally defined as multiple factors $(7,8)$. The factors include abnormalities inherited from the father (paternal abnormalities), fetal anatomical abnormalities, the age of the mother during the pregnancy, abortion history and environmental risk factors, such as caffeine, alcohol, cigarettes. In addition, factors such as genetics, infection, hematology and autoimmunity have been previously identified as important for the occurrence of miscarriages $(5,7,8)$. The pathogenesis of EPL remains to be elucidated (2). Previous studies based on advanced molecular genetics methods have investigated EPL pathogenesis. The development of the human placenta is critical for embryonic development and successful pregnancy. One of the important factors for the development and function of the placenta is epigenetic mechanism in gene expression $(3,9)$. Epigenetic factors are defined as DNA methylation $(\mathrm{CpG})$, chromatin histone modification and noncoding regulatory RNAs (8). The growth and progress of a fetus depend on the placental function and vein development; therefore, placental samples may be used for genomic and epigenetic research in miscarriage cases (4). MicroRNAs (miRNAs) have been identified as, the important molecules in genetic and genomic-based 
studies. It has been estimated that there are $>2,000$ miRNAs in humans, which are single stranded RNA molecules with 20-nucleotide length, which are not protein coding (9). One miRNA may regulate 200 mRNA functions. miRNA expression levels may change from one tissue type to another and also in different diseases. It has been previously established that miRNAs contribute to various biological events, such as development, differentiation, fertility and canceration (10-12). A previous study summarized that abnormal miRNA expression may be associated with $\sim 400$ diseases to human diseases (13). During pregnancy miRNA expression profiles in placenta and maternal blood have an important epigenetic role during the growth and development of fetus $(14,15)$. Additionally, miRNA expression in the placenta is regulated by environmental factors, signaling pathways and epigenetic modifications (16). There are three aims for genetics/genomics researches when investigating miscarriage patients. Firstly, to determine a DNA/RNA-based biomarker which may determine the direct predictive value of a couple's risk for miscarriage. Secondly, to establish gene/protein expression profiles, pathways and connections, which occur at the (un)successful establishment of pregnancy. Thirdly, apply hypothesis-based and hypothesis-free studies to pinpoint loci coding for novel non-invasive biomarkers applicable in clinical conditions for early pregnancy complications (8). Fetal nucleic acids that occur in the maternal plasma and serum are used by specific isolation methods for early diagnosis of genetic-based diseases such as preeclampsia, detection of fetal gender in women with a high risk of gender-associated disorders, fetal Rhesus ( $\mathrm{Rh}$ ) status and trisomies in pregnancies with a high risk for hemolytic disease of newborn (HDN) $(9,17,18)$. Fetal nucleic acids and miRNAs in maternal blood are analyzed by advanced molecular methods, such as quantitative polymerase chain reaction (qPCR), DNA/RNA microarrays and sequencing methods (18).

The miRNA microarray findings in the present study revealed that 4 miRNAs, hsa-miR-125a-3p, hsa-miR-3663-3p, hsa-miR-423-5p and hsa-miR-575, were upregulated in tissue samples. In maternal plasma, 2 miRNAs (hsa-let-7c, hsa-miR-122) were upregulated and one miRNA (hsa-miR-135a) was downregulated. A total of 6 out of 7 dysregulated miRNAs were validated by reverse transcription (RT)-qPCR. The target genes of these dysregulated miRNAs were identified using the GeneSpring database. The present study aimed to quantify miRNA expression levels in villous tissue and maternal plasma in EPL cases, to determine the miRNAs which exhibit changes in expression levels, and to detect the target genes of dysregulated miRNAs and the associated pathways which may establishing the association between miRNAs and the factors leading to EPL.

\section{Materials and methods}

Sample processing. Villous tissue samples which produce placenta and blood were collected from a total of 24 patients, 16 patients with EPL in 6th-8th gestational weeks (GWs) and 8 abortion cases (control group) in 6th-8th GW. Samples were collected from Department of Obstetrics and Gynecology, Cerrahpasa Medical Faculty, Istanbul University (Istanbul, Turkey) and Medicus Health Center (Istanbul, Turkey) between
January 2013 and May 2015. All patients and volunteers have been asked to sign written informed consent, the present study was also approved by the Ethical Committee. All informed consent forms are documented with the signature of the patients. Women in the control group had requested a surgical termination of pregnancy for non-medical reasons, and women in the EPL group had undergone surgical uterine evacuation. Villous tissue samples were collected immediately after the surgical procedure in sterile collection containers with physiological saline solution and were stored at $-80^{\circ} \mathrm{C}$ until used. The blood samples were collected immediately prior the surgical procedure in ethylenediaminetetraacetic acid (EDTA) -containing tubes (BD Biosciences, Franklin Lakes, NJ, USA) and immediately centrifuged at $1,400 \times \mathrm{g}$ for $10 \mathrm{~min}$ at $4^{\circ} \mathrm{C}$ to separate the plasma. Plasma samples were carefully transferred into new tubes and stored at $-80^{\circ} \mathrm{C}$ until used. Samples were stored in Istanbul University, Department of Molecular Biology and Genetics for experimental research.

The present study was divided into two stages: i) MiRNA profiling of villous samples and maternal plasma using microarrays; and ii) validation of dysregulated miRNAs using RT-qPCR (Fig. 1).

Total RNA isolation from villous tissue samples. Total RNA, including miRNAs was extracted from the villous tissue samples with the mirVana miRNA Isolation kit (Ambion; Thermo Fisher Scientific, Inc., Waltham, MA, USA) according to the manufacturer's protocol. Total RNA was eluted with $50 \mu \mathrm{l}$ mirVana elution solution. The quantity and quality of obtained total RNA was determined using NanoDrop ND-2000 spectrophotometer (Thermo Fisher Scientific, Inc.) and with the Agilent RNA Nano Chip kit on Agilent Bioanalyzer 2100 system (Agilent Technologies, Inc., Santa Clara, CA, USA). High quality (RNA integrity number $\geq 7$ ) samples from isolated total RNA were selected for microarray analysis. The isolated total RNAs were stored at $-80^{\circ} \mathrm{C}$ during laboratory preparation.

Total RNA isolation from maternal plasma samples. Total RNA, including miRNAs was isolated from $500 \mu \mathrm{l}$ maternal plasma sample using the mirVana miRNA Isolation kit (Ambion; Thermo Fisher Scientific, Inc.) according to the manufacturer's protocol. Synthetic Caenorhabditis elegans (C. elegans) miRNA, cel-miR-39 (working solution $1.6 \times 10^{8}$ copies/ $\mu$; Qiagen miRNeasy Serum/Plasma Spike-in control Qiagen, Inc., Valencia, CA, USA), was spiked into each sample (following the addition of a lysis buffer) to determine the purification quality and to normalize variation. Synthetic cel-miR-39 was selected as a spike-in due to the absence of homologous sequences in humans. Total RNA was eluted with $30 \mu \mathrm{l}$ mirVana elution solution. The quantity total RNA obtained was determined using NanoDrop ND-2000 spectrophotometer (Thermo Fisher Scientific, Inc.) and subsequently by the Agilent Small RNA Chip kit on Agilent Bioanalyzer 2100 system (Agilent Technologies, Inc.). The isolated total RNA was stored at $-80^{\circ} \mathrm{C}$ during laboratory preparation.

miRNA microarray analysis. In total, 32 EPL samples which were 16 maternal plasma and 16 villous tissue, furthermore 16 normal samples which were 8 maternal plasma and 8 villous 


\section{miRNAs Screening \& Validation studies}

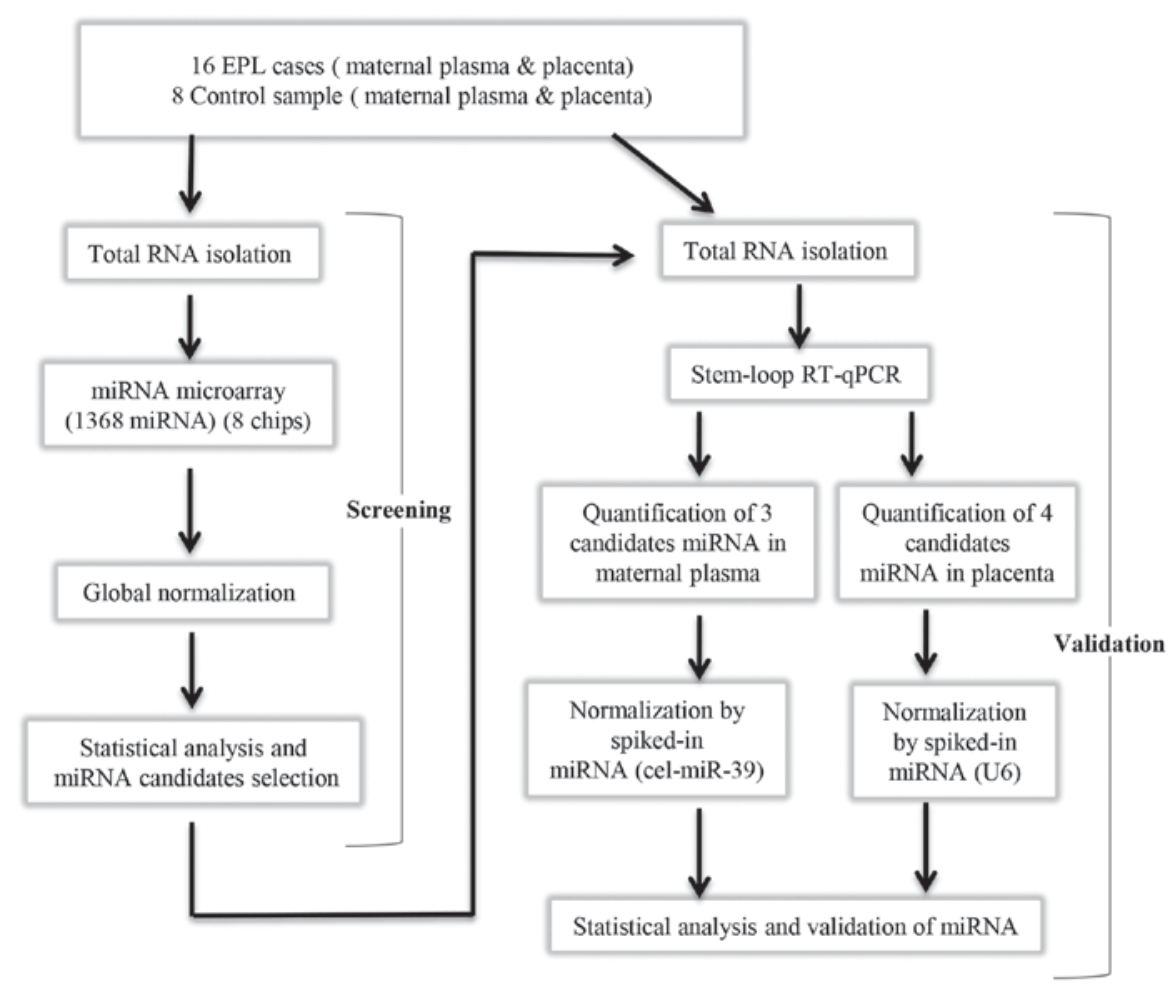

Figure 1. Schematic of the investigation process of the present study of quantification of miRNA profiles of $16 \mathrm{EPL}$ vs. 8 control placenta and maternal plasma samples. A total of 1,368 miRNAs were screened using microarray and 7 dysregulated miRNAs were subsequently validated by stem-loop RT-qPCR in tissues and maternal plasma. EPL, early pregnancy loss; miRNA, microRNA; RT-qPCR, reverse transcription-quantitative polymerase chain reaction.

tissue were analyzed by Agilent miRNA microarray chips (G4870A SurePrint G3 Human v16 miRNA 8x60K microarray; Agilent Technologies, Inc.). The miRNA Labeling and Hybridization kit (Agilent Technologies, Inc.) was used according to the manufacturer's protocol. Total RNA used for each villous sample was $100 \mathrm{ng} / \mu \mathrm{l}$ and maternal plasma sample of $\sim 50 \mathrm{ng} / \mu \mathrm{l}$ RNA (which included small RNAs). Each sample was labeled with cyanine 3-cytidine bisphosphate (pCp-Cy3) and the labeled RNA was hybridized at $55^{\circ} \mathrm{C}$ for $24 \mathrm{~h}$ to Human miRNA Microarray version 16 (Agilent Technologies, Inc.) slides, which included 1,368 miRNAs encoded by genes located across all chromosomes. After washing the microarray slides with Gene Expression Wash buffers (Agilent Technologies, Inc.) in order to increase the stringency, the slides were scanned by an Agilent SureScan Microarray scanner (model G2600D).

Agilent Feature Extraction version 11.0 was used for image analysis. The raw data was statistically analyzed using GeneSpring GX version 12.6 (Agilent Technologies, Inc.). In this database, the raw data produced was normalized by quantile normalization and probes with $<50 \%$ coefficient of variation were filtered. From these probes, a Student's t-test with a Bonferroni correction for the familywise error rate was performed and the following thresholds were used $\mathrm{P}<0.05$ and fold-change (FC) $>2$ were identified to be significantly dysregulated. The dysregulated miRNA results were obtained from the GeneSpring database (19) and gene targets and pathways associated with these genes have been listed in the present study according to this database. The target genes of significantly differentially expressed miRNAs were predicted and pathway analysis in villous and maternal plasma samples was performed using GeneSpring version 12.6, a nominal $\mathrm{P}<0.05$ was used to select significant pathways. RefSeq Protein ID, RefSeq Transcript ID, Entrez Gene ID, and UniGene ID annotations were selected for the present study experiment. Pathway sources used in the analysis, included WikiPathways-Analysis, WikiPathways-Reactome, and WikiPathways-Other, which are valid for Homo sapiens. The results of the pathway analysis were obtained from the 'Single Experiment Analysis (SEA)' heading in the 'GeneSpring' database. Calculation of the P-value is indicated in the subsequent formula:

$$
P(X \geq k)=\frac{1}{\left(\begin{array}{l}
u \\
n
\end{array}\right)} \sum_{i=k}^{i \operatorname{Max}}\left(\begin{array}{c}
m \\
i
\end{array}\right)\left(\begin{array}{c}
u-m \\
n-i
\end{array}\right)
$$

Where $\mathrm{u}$ stands for the total number of entities in the technology which have same ID for annotations selected in SEA; $m$, number of entities in the entity list which have same ID for the annotation selected; n, number of pathway entities that matched with the technology; and $\mathrm{k}$, number of pathway entities that matched with the entity list.

miRNA validation by stem-loop RT-qPCR. The validation of miRNA microarray results on the EPL and control in maternal plasma and tissue samples was performed using RT-qPCR. Dysregulated miRNAs $(\mathrm{P}<0.05$ and $\mathrm{FC}>2)$ obtained from the microarray bioinformatics analysis were subsequently validated in 24 (16 EPL \& 8 control) tissue samples and 24 (16 
EPL \& 8 control) maternal plasma samples using stem-loop RT-qPCR.

The present study synthetic cel-miR-39 for normalization and detection of extraction efficiency in maternal plasma, and U6 for normalization in tissue samples of TaqMan RT-qPCR in dysregulated miRNAs. miRNAs from each sample were reverse-transcribed into cDNA using TaqMan microRNA Reverse Transcription kit (Thermo Fisher Scientific, Inc.) and miRNA specific 5X stem-loop primers (TaqMan $^{\mathrm{TM}}$ MicroRNA Assay; Applied Biosystems; Thermo Fisher Scientific, Inc.) hsa-miR-125a-3p (cat. no. 4427975, RT: 002199), hsa-miR-3663-3p (cat. no. 4427975; RT: 465775_mat), hsa-miR-575 (cat. no. 4427975; RT: 001617), hsa-miR-425-5p (cat. no. 4427975; RT: 002302), has-let-7c (cat. no. 4427975; RT: 000379), hsa-miR-122 (cat. no. 4427975; RT: 002245), hsa-miR-135a (cat. no. 4427975; RT: 000460) in total reaction volume of $15 \mu \mathrm{l}$ according to the manufacturer's protocol. Transcription reaction was preformed using an 8800 PCR thermal cycler system (Agilent Technologies, Inc.) by heating to $16^{\circ} \mathrm{C}$ for $30 \mathrm{~min}$, followed by $42^{\circ} \mathrm{C}$ for $60 \mathrm{~min}$ and $85^{\circ} \mathrm{C}$ for $5 \mathrm{~min}$. The qPCR was performed using TaqMan miRNA qPCR Universal PCR Master mix assay (Thermo Fisher Scientific, Inc.), and 20X primer (Thermo Fisher Scientific, Inc.), which included fluorescein dye-specific probes for each previously stated miRNA in a $20 \mu 1$ total reaction mixture. Every batch of amplifications included two water blanks and primers as no template negative controls for each cDNA product and qPCR steps. The following thermocycling conditions were used for the PCR: $50^{\circ} \mathrm{C}$ for $2 \mathrm{~min}, 95^{\circ} \mathrm{C}$ for $10 \mathrm{~min}$; 40 cycles of $95^{\circ} \mathrm{C}$ for $15 \mathrm{sec}$ and $60^{\circ} \mathrm{C}$ for 1 mins. The qPCR was replicated 2 times for each sample. RT-qPCR results were analyzed as follows: $\Delta \mathrm{Ct}=\mathrm{Ct}$ sample-Ct cel-miR-39; $\Delta \Delta \mathrm{Ct}=\Delta \mathrm{Ct}$ case $-\Delta \mathrm{Ct}$ control. Finally, the $\Delta \Delta \mathrm{Ct}$ was normalized against cel-miR-39. All reactions were analyzed by using the $2^{-\Delta \Delta C t}$ method (20).

\section{Results}

Characteristics of samples. There were two experimental groups in the present study, including the patient group (the mean pregnancy age is $\sim 30$ ) and the control group (the mean pregnancy age is 29). In both groups, none of the participants had anatomic abnormalities, infections, endocrine or metabolic disorders, autoimmune diseases, paternal or maternal chromosomal abnormalities, or smoked. Table I presents the characteristics of the two experimental groups.

miRNA microarray results. Statistical analysis of microarray results in maternal plasma identified 3 dysregulated miRNAs. From the three miRNAs in maternal plasma samples two were upregulated and one was downregulated (Table II). Expression levels for each miRNA in maternal plasma have been presented as box-whisker plots (Fig. 2) and hierarchical clustering analysis revealed that the subgroups were well-differentiated from the unified set of differentially expressed miRNAs (Fig. 3). In the villous tissue samples, all 4 miRNAs were upregulated (Table III) and have been found to be significantly dysregulated $(\mathrm{P}<0.05$ and $\mathrm{FC}>2)$ in the EPL group compared with the control group. In villous samples dysregulated miRNAs have
Table I. Clinical summary of EPL and normal pregnancy control groups.

\begin{tabular}{lcccc}
\hline Age & $\begin{array}{c}\text { Mean } \\
\text { gravidity } \\
\text { (orders) }\end{array}$ & $\begin{array}{c}\text { Mean of } \\
\text { miscarriage }\end{array}$ & $\begin{array}{c}\text { Gestational } \\
\text { age (weeks) }\end{array}$ \\
\hline EPL & $30.23 \pm 5.81$ & 1.81 & 2.75 & $7.22 \pm 2.8$ \\
Control & $28.62 \pm 5.24$ & 2.12 & 0 & $7.11 \pm 5.2$ \\
\hline
\end{tabular}

EPL, early pregnancy loss.

been represented as box-whisker plots (Fig. 4) and hierarchical clustering (Fig. 5).

Stem-loop RT-qPCR findings. Stem-loop RT-qPCR was performed to validate the 7 differentially expressed miRNAs identified in the miRNA microarray analysis. A total of 24 plasma samples, consisting of 16 EPL maternal plasma and 8 normal plasma samples, were used for maternal plasma validation and the same quantity of EPL and normal villous tissues were used for validation by stem-loop RT-qPCR. The present study identified 3 miRNAs (hsa-let-7c, hsa-miR-122 and hsa-miR-135a) in maternal plasma samples and 3 miRNAs, including hsa-miR-125a-3p, hsa-miR-3663-3p and hsa-miR-575 in villous samples were validated to be upregulated in EPL samples. However, miR-425-5p did not demonstrate any marked expression alterations in villous tissues by RT-qPCR. Consistent with miRNA microarray analysis, the changes of all 6 upregulated miRNAs in EPL and control samples in villous tissues and maternal plasma are presented in Table IV and Fig. 6.

Target genes and pathway analysis. Target analysis for validated dysregulated miRNAs in GeneSpring version 12.6 identified 432 target genes for the villous sample dysregulated miRNAs and 274 target genes for maternal plasma dysregulated miRNAs, where $\mathrm{P}<0.05$. Pathway analysis of targeted genes of villous sample and maternal plasma samples with $\mathrm{P}<0.05$ by GeneSpring identified 233 and 130 pathways, respectively. The top significant pathways (sorted by lowest P-value) of the target genes was predicted by differentially expressed miRNAs in villous samples and maternal plasma are presented in Tables V and VI, respectively.

\section{Discussion}

The formation of placenta is of high importance in a healthy pregnancy process and in embryo development. Mechanisms such as differentiation, migration, invasion, angiogenesis, proliferation and apoptosis have a significant role in placenta formation $(8,9)$. It has been estimated that $>500$ miRNA expression levels specific to placenta have been identified during pregnancy. The expression levels of these miRNAs are regulated by environmental factors such as hypoxia, signaling pathways and epigenetic modifications $(9,12)$. Abnormal expression levels of miRNAs were also identified in gynecological diseases, including ovarian 
Table II. Dysregulated miRNAs for early pregnancy loss and control maternal plasma samples. Corrected P<0.05 and FC $>2$.

\begin{tabular}{lccccc}
\hline Systematic name & Regulation & P-value & FC & Active sequence $\left(5^{\prime} \rightarrow 3^{\prime}\right)$ & Chromosome \\
\hline hsa-let-7c & Down & 0.0041 & -5.16 & AACCATACAACCTACTACC \\
hsa-miR-122 & Up & 0.00039 & 4.72 & CAAACACCATTGTCACACT & 21 \\
hsa-miR-135a & Up & 0.00294 & 2.62 & CGCCACGGCTCCA & 3
\end{tabular}

FC, fold-change; miRNA, microRNA.
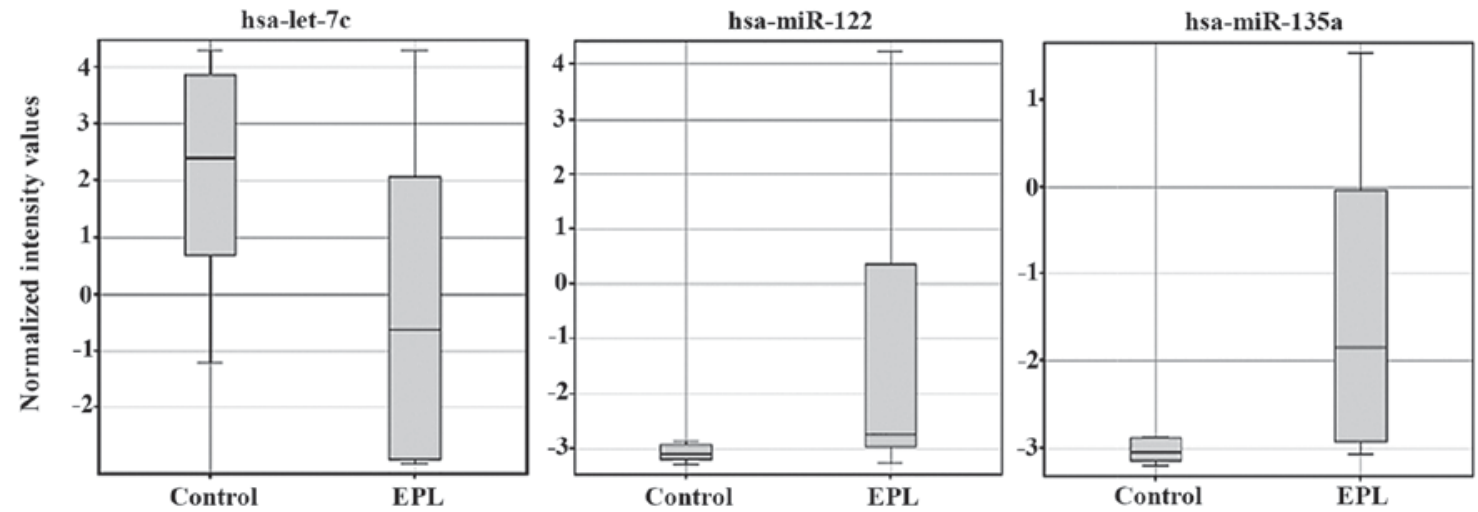

Figure 2. Box-whisker plots of 3 dysregulated miRNAs in maternal plasma from 24 samples, 16 EPL and 8 controls detected by microarray techniques. miRNA/miR, microRNA; EPL, early pregnancy loss.

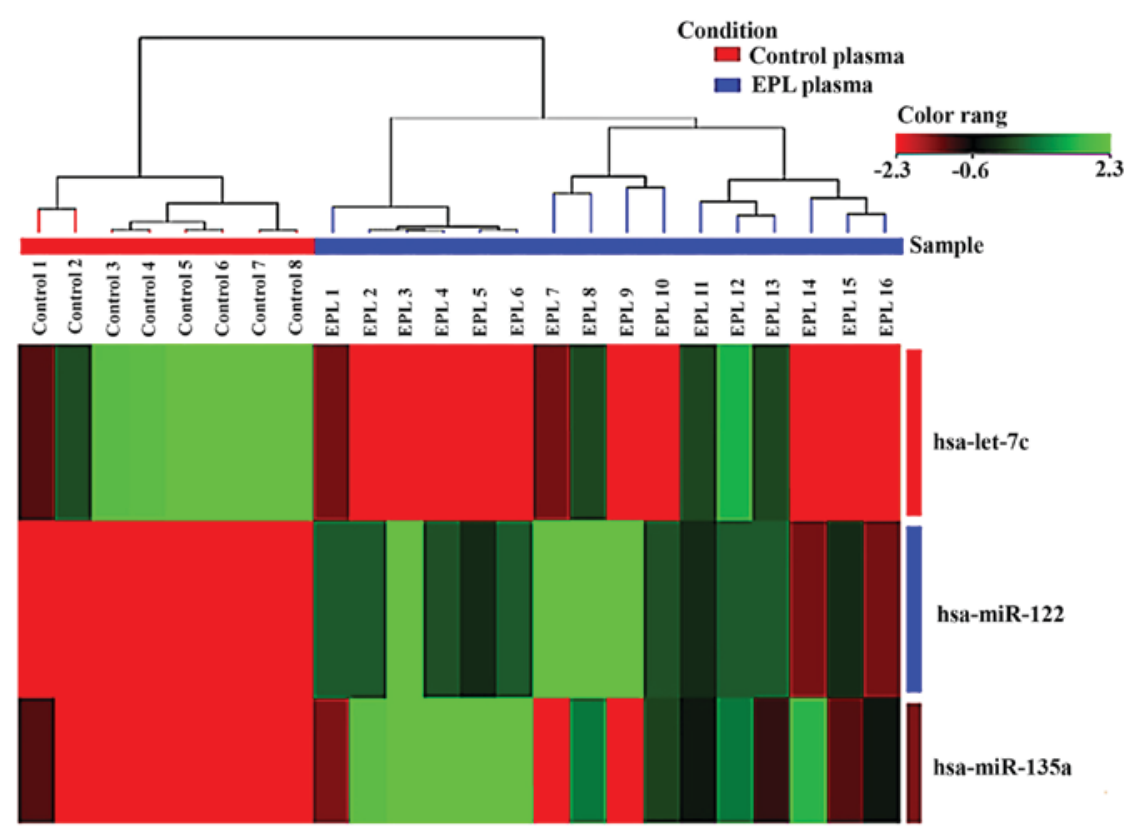

Figure 3. Hierarchical clustering of the 3 dysregulated miRNAs in maternal plasma samples using microarray techniques. The sample clustering tree appears at the top. Green indicates high expression and red low expression. miRNA/miR, microRNA; EPL, early pregnancy loss.

carcinoma, endometriosis, benign gynecological conditions and infertility (21). In the present study, maternal blood and villous samples, which have an important role in the formation of placenta were collected from EPL cases and patients voluntarily having abortion. MiRNA expressions were quantified in the samples. In EPL patients, significant miRNA expression changes were identified compared to the control patients. According to the microarray findings, a total of 4 miRNAs were identified in the tissue samples (Table III) and 4 miRNAs in maternal plasma samples (Table II) with evident expression differentiations. These findings were confirmed for 7 miRNAs, using RT-qPCR as presented in Table IV. MiR-125a-3p, miR-3663-3p, miR-575, let-7c, miR-122 and hsa-miR-135a obtained from villous 
Table III. Dysregulated miRNAs for early pregnancy loss and control villous sample Corrected $\mathrm{P}<0.05$ and $\mathrm{FC}>2$.

\begin{tabular}{lccclc}
\hline Systematic name & Regulation & P-value & FC & Active sequence $\left(5^{\prime} \rightarrow 3^{\prime}\right)$ & Chromosome \\
\hline hsa-miR-125a-3p & Up & 0.000407 & 2.15 & GGCTCCCAAGAACCTCA & 19 \\
hsa-miR-3663-3p & & 0.000337 & 2.73 & GCGCCCGGCCT & 10 \\
hsa-miR-423-5p & & 0.0332 & 2.01 & AAAGTCTCGCTCTCTG & 17 \\
hsa-miR-575 & & 0.00275 & 2.33 & GCTCCTGTCCAACTGGCT & 4 \\
\hline
\end{tabular}
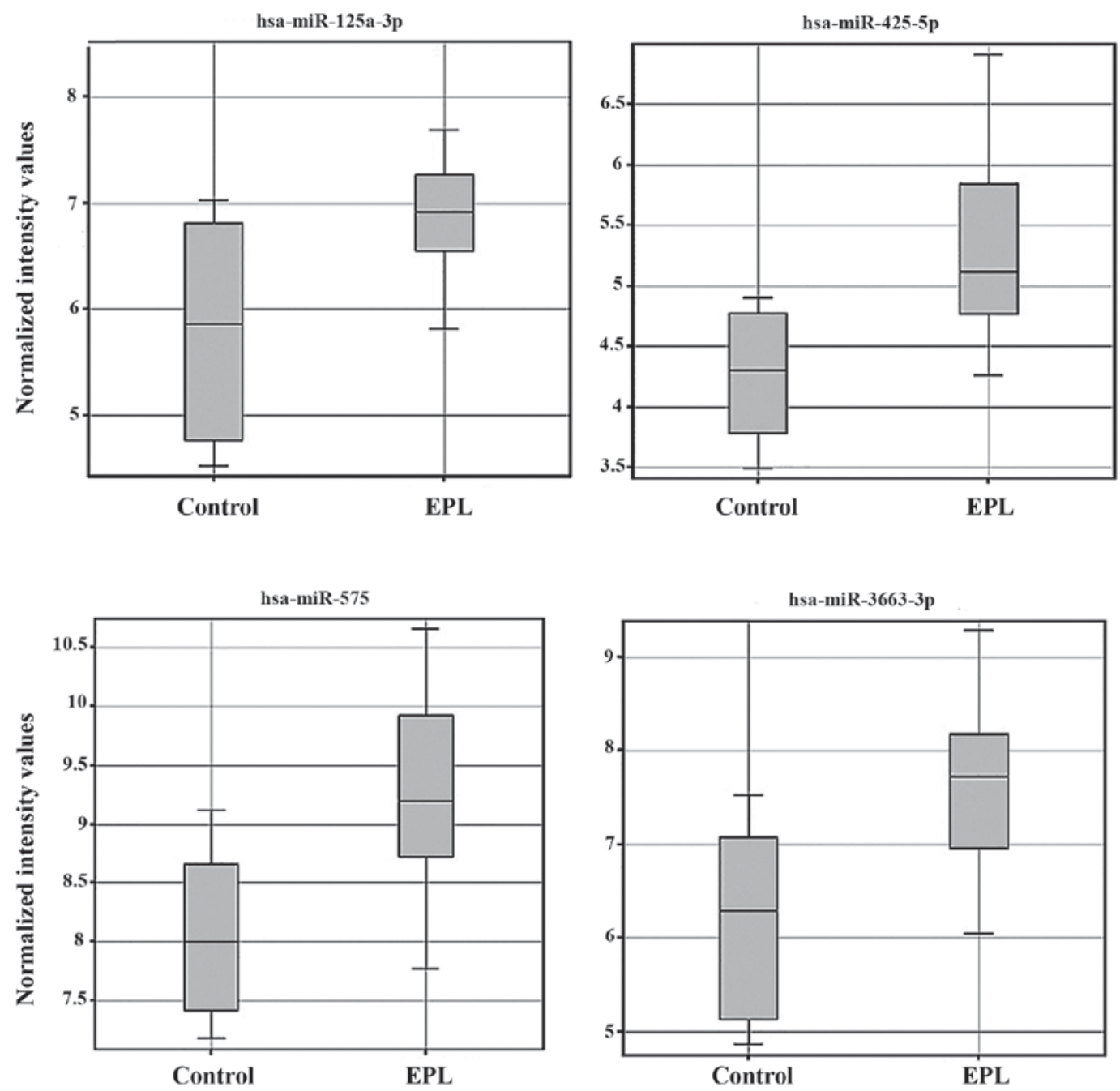

Figure 4. Box-whisker plots of 4 dysregulated miRNAs in villous samples from 24 samples, 16 EPL and 8 controls, detected by microarray techniques $\mathrm{miRNA} / \mathrm{miR}$, microRNA; EPL, early pregnancy loss.

and maternal plasma samples have been validated following RT-qPCR analysis. In the present study EPL cases between 6th-8th GWs, for which the reason for the occurrence of the EPL were unknown, have been investigated. Tissue and blood samples have been collected from each subject and miRNA analyses were performed for each case. To the best of our knowledge, based on EPL grouping and the performed analyses, the present study may be defined as first in this area.

Dysregulated miRNAs obtained from villous tissue samples. The comparison between EPL and control group samples by microarray and RT-qPCR revealed that miR-125a-3p, miR-3663-3p and miR-575 were significantly upregulated in tissue samples.

The miR-125 family is consists of miR-125a, miR-125b-1 and miR-125-2 homologues which have a wide variety of functions, such as control of proliferation of the hematopoietic stem cells, control of genes associated with carcinomas, whose expression patterns are protected in the genome $(22,23)$. The present study identified that expression of miR-125a-3p, which is the mature form of miR-125a, was increased in villous tissue samples of EPL group compared with the control. Li and Li demonstrated that miR-125a expression was increased in Unexplained 


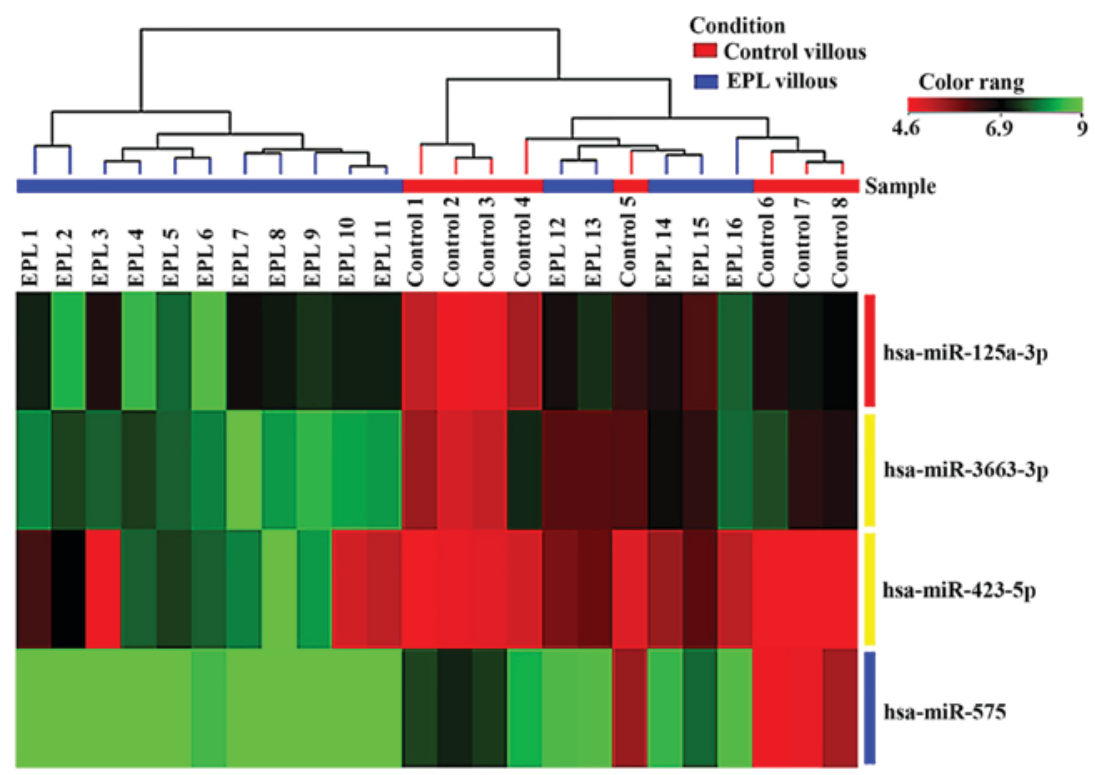

Figure 5. Hierarchical clustering of 4 dysregulated miRNAs in villous samples by microarray techniques. Green indicates high expression and red low expression. miRNA/miR, microRNA; EPL, early pregnancy loss.

Recurrent Spontaneous Abortion (URSA) samples compared with control samples (22). Therefore, miR-125a expression may be associated with recurrent miscarriages (24). A previous study revealed that expression levels of ErbB2 receptor tyrosine kinase 2 (ERBB2), which is the receptor of heparin-binding epidermal growth factor and leukemia inhibitory factor receptor $(L I F R)$, and leukemia inhibitory factor have an effect on the implantation of embryo on the uterus wall which is increased in implantation stage of menstrual cycle (25). ERBB2 and LIFR are among the genes targeted by miR-125a-3p, therefore, increase in miR-125a-3p may lead to a decrease in the expression level of ERBB2 and LIFR genes at the implantation stage. It is possible that a reduced expression level of these genes at the implantation stage may negatively affect the implantation process. Ninio-Many et al revealed that increase in miR-125a-3p expression prevents the expression of the Fyn gene which is part of the Scr kinase family, which may lead to reduced cell adhesion, proliferation, migration and viability (26). Along with the decrease of Fyn gene expression, the expression levels of focal adhesion kinase $(F A K)$, paxillin and V-Akt murine thymoma viral oncogene $(A k t)$ genes located downstream of Fyn were also decreased. Subsequently, this limits the viability and mobility of cells and blocks the cell cycle (26). The present study identified that increased miR-125a-3p expression may lead to a miscarriage by reducing the expression levels of $F A K$, paxillin and Akt genes during adhesion, proliferation and migration processes which are required for placenta and embryo development. Zhang et al observed that miR-125a-3p expression has been increased and expression levels of metastasis-associated gene 1 (MTAl), which has a critical role in chromatin remodeling and histone deacetylase complexes in cells, has been reduced in the non-small cell lung cancer (27). Increased expression of miR-125a in breast cancer cells suppresses the growth of cells by reducing the expression of HuR protein which is a stress-induced RNA binding protein (28). miR-125a binds to the 3'-untranslated region (UTR) of the gene encoding the transcription factor estrogen-related receptor a in squamous cell carcinoma of the mouth and reduces the expression of this gene, and thereby suppressing the cell proliferation and increasing apoptosis (29). Considering these previous findings, cell migration, proliferation and cell adhesion are suppressed, and apoptosis is increased due to the increased expression of miR-125a-3p in the cell. The absence of migration and adhesion, and excessive apoptosis during the development of the embryo in the cellular dimensions may lead to EPL. According to the Target Scan database, vascular endothelial growth factors (VEGFs) are target genes of miR-125-3p, which are important for placenta development. Choi et al investigated the correlation between recurrent miscarriages and decrease in the expression level of VEGFs, which are effective in angiogenesis (30). The present study determined that miR-125a expression increased in the villous trophoblast, which may lead to reduced VEGFs expression levels and lead to EPL by adversely affecting angiogenesis.

The present study identified miR-575 expression to be increased in tissue samples. Gu et al determined that expression level of miR-575 was increased in maternal serums in neural tube defects (NTDs) (31). It was previously observed that miRNA expression levels were severely reduced in serum within $24 \mathrm{~h}$ after birth. Therefore, it is possible that these miRNAs associated with the pregnancy may be used for the early diagnosis of NTD fetuses (31). Nardelli et al determined that miR-575 was expressed only in pregnant obese patients (32). Expression of this miRNA was demonstrated to be effective in the adipocytokine and mitogen-activated protein kinase signaling pathways which have an important role in placental development, leading to a decrease in the expression of adiponectin receptor 2 and solute carrier family 2 member 1 proteins and epigenetic regulation of placental metabolism (32). Based on the findings obtained in the current study, this may contribute to EPL as degradation of the adipocytokine and mitogen-activated protein kinase signal pathways disrupts the placental development and function, as a 
Table IV. Differential expression of validated signature miRNAs for early pregnancy loss and control samples using microarray and TaqMan RT-qPCR platforms.

\begin{tabular}{|c|c|c|c|c|c|c|}
\hline \multirow[b]{2}{*}{ Sample } & \multirow[b]{2}{*}{ Regulation } & \multirow[b]{2}{*}{ miRNA } & \multicolumn{2}{|c|}{ miRNA microarray } & \multicolumn{2}{|c|}{ RT-qPCR } \\
\hline & & & $\mathrm{FC}$ & P-value & $\mathrm{FC}$ & P-value \\
\hline \multirow[t]{3}{*}{ Maternal plasma } & Up & hsa-miR-122 & 4.7 & 0.00039 & 1.57 & 0.0241 \\
\hline & & hsa-miR-135a & 2.62 & 0.00294 & 3.8 & 0.0249 \\
\hline & Down & hsa-let-7c & -5.16 & 0.00412 & -2.68 & 0.0323 \\
\hline \multirow[t]{4}{*}{ Villous tissue } & Up & hsa-miR-125a-3p & 2.15 & 0.000407 & 1.72 & 0.0258 \\
\hline & & hsa-miR-3663-3p & 2.73 & 0.000337 & 2.02 & 0.0075 \\
\hline & & hsa-miR-423-5p & 2.01 & 0.03323 & $\mathrm{~N}$ & $\mathrm{~N}$ \\
\hline & & hsa-miR-575 & 2.33 & 0.000275 & 2.5 & 0.00029 \\
\hline
\end{tabular}

FC, fold-change; N, no result; miRNA, microRNA; RT-qPCR, reverse transcription-quantitative polymerase chain reaction.

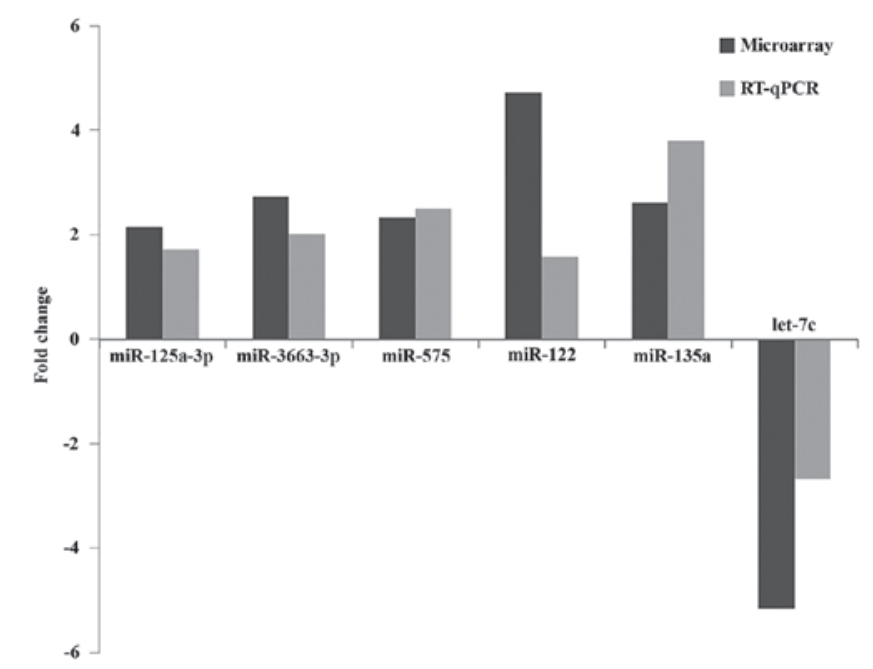

Figure 6. Comparison of validated miRNA expression levels between the microarray and TaqMan RT-qPCR platforms in both maternal plasma and villous samples, separately. 3 miRNAs (hsa-let-7c, hsa-miR-122 and hsa-miR-135a) were validated in maternal plasma samples and 3 miRNAs, including hsa-miR-125a-3p, hsa-miR-3663-3p and hsa-miR-575 were validated in villous samples. RT-qPCR, reverse transcription-quantitative polymerase chain reaction; miRNA/miR, microRNA.

result of the increased expression of miR-575. It was previously identified that the increased expression of miR-575 in breast cancer cells may lead to the expression of phosphatidylinositol specific phospholipase $\mathrm{CX}$ domain containing 1 to reduce and thus to suppress cell proliferation (33). The present study demonstrated that increased expression of this miRNA may lead to EPL by having diverse effects on important processes, such as cell growth and proliferation in the development of placenta and embryo.

Previous studies on miR-3663-3p have revealed that its expression was elevated in particular types of cancer, such as primary cutaneous melanoma (34), nasopharyngeal carcinoma (35) and prostate cancer (36). The present study determined that the expression in the miRNA scans of the tissue samples obtained from the EPL cases was increased when compared with normal tissues. However, as is no specific research on this miRNA, only target genes and their associated functions could be obtained following bioinformatics analysis. The genes targeted by miR-3663-3p were found to be effective in differentiation processes, cell division, apoptosis and cell proliferation (37-39). Pathway analysis for predicted target genes of the validated miRNAs in tissue samples (miR-125a-3p, miR-3663-3p and miR-575), including epidermal growth factor receptor $(E G F R)$, T cell receptor, ErbB, Wnt, mitogen activated protein kinase (MAPK), interleukin (IL)-2,-3,-5 and -7, revealed signaling pathways involved may be apoptosis, oxidative stress, focal adhesion and janus kinase-signal transducer and activator of transcription (JAK-STAT) pathways (Table V) which have previously been identified to be associated with miscarriage pathogenesis $(2,3,24,40-43)$.

Dysregulated miRNAs obtained from maternal plasma samples. Previous studies on maternal plasma miRNAs use biomarkers for reproductive diseases, specifically for EPL and URSA, are limited (44-46). To the best of our knowledge the present study is the first to investigate circulating miRNAs in EPL patients. The comparison of EPL and control group samples using microarray and RT-qPCR techniques revealed that miR-135a, miR-122 and let-7c were significantly dysregulated in maternal plasma samples.

Expression level of miR-135a was determined to be higher in the maternal plasma samples. Petracco et al detected increased expression of miR-135a in endometriosis endometrium tissue samples during the menstrual cycle proliferation and luteal phases and reduced in the ovulation phase (47). It is possible that binding of miR-135a to the 3'-UTR region of HOXA10 gene may be increased along with the reduced expression of HOXA10, and may lead to EPL by adversely affecting the uterus development and embryo implantation. In a previous study conducted on lung cancer, it was determined that miR-135a acted as a tumor suppressor (47). It is possible that binding of miR-135a to the 3'-UTR region of HOXA10 gene may be increased along with the reduced expression of HOXA10, and may lead to EPL by adversely affecting the uterus development and embryo implantation. In a previous study conducted on lung cancer, it was determined that miR-135a acted as a tumor suppressor (48). It was also determined that miR-135a reduced the expression of Kruppel-like 
Table V. Top pathways involved by number of genes targeted by dysregulated miRNAs, including miR-125a-3p, miR-3663-3p and miR-575, obtained from villous samples.

\begin{tabular}{|c|c|c|c|}
\hline Pathway & Nominal P-value & Matched entities & Pathway entities \\
\hline Hs EGF EGFR signaling pathway WP437-72106 & 0.000000001015 & 7 & 162 \\
\hline Hs TSLP signaling pathway WP2203-72104 & 0.0000003804 & 3 & 47 \\
\hline Hs T cell receptor and co-stimulatory signaling WP2583-75360 & 0.0000003804 & 3 & 30 \\
\hline Hs ErbB signaling pathway WP673-69914 & 0.0000003804 & 3 & 54 \\
\hline Hs apoptosis WP254-72110 & 0.0000003804 & 3 & 84 \\
\hline Hs calcium regulation in the cardiac cell WP536-75219 & 0.000001417 & 6 & 150 \\
\hline Hs IL-7 signaling pathway WP205-70018 & 0.00005262 & 2 & 27 \\
\hline Hs MAPK cascade WP422-72129a & 0.00005262 & 2 & 29 \\
\hline Hs IL-2 signaling pathway WP49-75383 & 0.00005262 & 2 & 42 \\
\hline Hs integrin-mediated cell adhesion WP185-71391 & 0.00005262 & 2 & 99 \\
\hline Hs Wnt signaling pathway and pluripotency WP399-74897ª & 0.00005262 & 2 & 100 \\
\hline Hs IL-3 signaling pathway WP286-72139 & 0.00005262 & 2 & 49 \\
\hline Hs focal adhesion WP306-71714 ${ }^{\mathrm{a}}$ & 0.0001974 & 5 & 188 \\
\hline Hs myometrial relaxation and contraction pathways WP289-72107 & 0.0001974 & 5 & 156 \\
\hline Hs MAPK signaling pathway WP382-72103 ${ }^{a}$ & 0.0027438 & 4 & 168 \\
\hline Hs GPCRs class A rhodopsin-like WP455-74420a & 0.0027438 & 4 & 261 \\
\hline Hs oxidative stress WP408-69029 & 0.007262 & 1 & 30 \\
\hline Hs IL-5 signaling pathway WP127-70017 & 0.007262 & 1 & 40 \\
\hline Hs JAK-STAT WP2593-74127 & 0.007262 & 1 & 44 \\
\hline
\end{tabular}

aPathways are common pathway in villous sample and maternal plasma sample obtained from pathway analysis. Matched entities, genes targeted by dysregulated miRNA; Pathway entities, total number of genes in the pathway.

Table VI. Top pathways involved by number of genes targeted by dysregulated miRNAs, including miR-122, miR-135a and let-7c, obtained from maternal plasma samples.

Pathway

Nominal P-value Matched entities Pathway entities

Hs calcium regulation in the cardiac cell WP536-75219

$\begin{array}{lll}0.00002112 & 2 & 150\end{array}$

Hs MAPK cascade WP422 -72129a

0.00002112

$2 \quad 29$

Hs inflammatory response pathway WP453-63217

0.00002112

2

0.00002112

33

Hs focal adhesion WP306-71714 ${ }^{\mathrm{a}}$

0.004421

188

Hs myometrial relaxation and contraction pathways WP289-72107

0.004632

Hs GPCRs, Class C Metabotropic glutamate, pheromone WP501-63205

0.004632

Hs Regulation of toll like receptor signaling pathway WP1449-72114

0.004632

Hs Toll-like receptor signaling pathway WP75-72133

Hs IL-1 signaling pathway WP195-74010

0.004632

Hs Wnt signaling pathway and pluripotency WP399-74897ª

0.004632

Hs JAK STAT WP2593-74127ª

0.004632

Hs MAPK signaling pathway WP382-72103 ${ }^{\mathrm{a}}$

0.009645

Hs Apoptosis Modulation and Signaling WP1772-63162

0.009645

Hs apoptosis WP254-72110a

0.009645

Hs GPCRs, class A rhodopsin-like WP455-74420a

0.009645

${ }^{a}$ Pathways are common pathway in villous sample and maternal plasma sample obtained from pathway analysis. Matched entities, genes targeted by dysregulated miRNA; Pathway entities, total number of genes in the pathway.

factor $8(K L F 8)$ which is a transcription factor, and suppressed the epithelial mesenchymal transition molecular mechanism of cancer cells, inhibiting cell migration and invasion (48). It believed that the increase in miR-135a expression may reduce 
the expression of $K L F 8$, leading to the prevention of migration and invasion processes, which are required during the embryo development, and may lead to EPL. Navarro et al detected that the increased levels of miRNA-135a in patients with classical Hodgkin's lymphoma may lead to increased apoptosis and suppression of cell proliferation, and that miR-135a may directly suppresses janus kinase 2 which is a cytoplasmic tyrosine kinase, followed by the suppression of the B cell lymphoma extra-large (Bcl-xL) gene which is an anti-apoptotic gene (49). These events lead to an increase in apoptosis and suppression in proliferation (49). The present study revealed that the expression of miR-135a increases significantly in the maternal plasma samples, leading to EPL by increasing apoptosis and inhibiting proliferation in the villous cells. In order for this miRNA to be used as a biomarker to identify EPL cases, the effects of the miR-135a on the genes that affect EPL require further investigation and treatment procedures for the disease must be specified according to the obtained results as a further step by investigating the targets of this miRNA. In this context, the present study may be used as the basis for biomarker research.

The present study identified that miR-122 expression levels were increased in the maternal plasma samples obtained from patients with EPL. The invasion of maternal decidua to placenta trophoblasts is the most important stage in the normal physiological course of a normal pregnancy, and apoptosis has a critical role in normal placental development (50). MiR-122 may be involved in apoptosis in cancer cells (51). A different previous study demonstrated that miR-122 increased the expression of oncogenes cyclin G1 (CCNG1) (52) and reduced the expression of a disintegrin and ADAM metallopeptidase domain 17, EGFR and FAK activation levels (53). Based on the data obtained from miR-122-associated cancer studies, it is possible that in the present study, the increased maternal plasma levels of this miRNA may have adversely affected the development of the embryo by reducing the expression of genes that are effective during migration, adhesion, invasion and apoptosis of placenta trophoblast cells and embryo cells in important stages as preimplantation and implantation in embryo development. Lasabová et al investigated the expression of miR-122, which was associated with apoptosis, and demonstrated an increased expression in the case of preeclampsia compared with normal placental samples (54). Therefore, considering the findings of the present study it is possible that the increased miR-122 expression in maternal plasma samples may lead to an inadequate level of apoptosis in the villous trophoblast cells that normally form the placenta during the developmental stage, which may to lead to EPL by affecting the development of placenta.

According to the present study, the expression level of let-7c, a member of the let-7 family was reduced in the maternal plasma samples from EPL patients compared with the control group. Previous studies have revealed that the let-7 family is associated with the cardiovascular system $(55,56)$. The important genes targeted by the let- 7 family, include toll-like receptor 4 (TLR4), oxidized low density lipoprotein receptor 1, $B c l-x l$ and argonaute-1 (AGOl) (56-58). It was previously determined that these genes are associated with the immune system, increased apoptosis, anti-apoptosis and miRNA biogenesis respectively. The genes targeted by the let-7 family have been identified as effective in embryonic stem cell and cardiovascular differentiation (59). Zhang et al determined the hypoxia-inducible factor $1 \alpha(H I F 1 \alpha) / A G O 1 / V E G F$, which are the factors related with let-7, have a role in the hypoxia-induced angiogenesis signaling pathway (51). HIFl $\alpha$ is a key transcription factor, which upregulates the expression levels of let-7, and let-7 targets $A G O 1$, leading to the translational suppression of $V E G F$ mRNA which has an important role in angiogenesis process (56). The anti-apoptosis protein Bcl-x1 which is affected by let-7c contributes to the apoptosis of epithelial cells and its expression is suppressed by let-7, leading to an increase in apoptosis. Reduced let-7c expression may lead to an increased expression of anti-apoptotic proteins such as Bcl-x1 and prevent apoptosis during development, which in turn may lead to miscarriage by adversely affecting the placental and embryonic development (60). According to the TargetScan database; Kruppel-like factor 9 (KLF9); CAMP responsive element binding protein $1(C R E B-1)$ and phosphatase and tensin homolog (PTEN) are let-7c target genes. KLF9 is a zinc-finger transcription factor that has a role in the regulation of estrogen and progesterone by modulating the activity of the progesterone receptor. Regulation of the KLF9 expression in the endometrium epithelial cells is important for embryo implantation in the menstrual cycle (57); therefore, it is believed that reduced expression of let-7c may lead to an increased expression of these gene and hormonal disturbances in the menstrual cycle. To the best of our knowledge, no research has been performed in this regard; however, the findings of the current study suggest that the decrease in the expression of let-7c may be associated with the role of KLF9 in endometrium implantation. Ventura et al demonstrated that $C R E B-1$ and PTEN were targeted by miR-17 and miR-19b which are expressed in the placenta during the early weeks of pregnancy, and that these miRNAs were associated with EPL, as they act as regulatory factors during placental development (4). A previous study revealed that expression of the PTEN gene in the villous trophoblast cells in the early stages of the placental formation occurred; however, this expression reduces with time (58). It was previously suggested that the increased expression of this gene in villous trophoblast cells may be associated with miscarriage (58). Several pathways have been identified from the pathway analysis for the predicted target genes of validated miRNAs in tissue samples preformed in the present study. The most important pathways which were investigated associated with miscarriage pathogenesis, included Wnt, IL-1, regulation of toll-like receptor and MAPK signaling as well as apoptosis, focal adhesion and JAK-STAT pathways (Table VI) $(2,3,24,41-43)$.

The primary aim of the present study was to demonstrate the significant difference in the expression of miRNAs between normal subjects and those with EPL by analyzing villous tissue and maternal blood samples. Subsequently, bioinformatics analysis of dysregulated miRNAs was also performed to detect targeted genes and their associated pathways. A total of 4 miRNAs in the tissue samples and 3 miRNAs in the maternal plasma had significant differences in expression according to the microarray results. The validation experiments have confirmed 6 miRNAs to be valid. It is possible to use the 3 miRNAs that have been detected in the maternal plasma as biomarkers for non-invasive prenatal diagnosis for this group of patients. It was 
previously determined that the genes targeted by the miRNAs detected in the villous tissue and maternal plasma samples contribute to in differentiation, development, cell division, proliferation, implantation and angiogenesis. Additionally, these processes are effective in embryo development; therefore, they are believed to be effective in the biological progression of the disease. Pathway analysis of the genes targeted by the validated miRNAs revealed that the associated pathways were frequently associated with miscarriage according to previous studies. It is possible that the detected miRNAs in the maternal plasma and tissue are involved in the EPL process. In order to take these findings one step further, future studies may validate the 3 miRNAs obtained from the maternal plasma screening in more patient samples in order for them to be used for early diagnosis. In addition, further studies should be designed on the target genes of the miRNAs detected in increased number of villous and maternal plasma samples from patients with EPL, and the associations of the miRNAs with the target mRNAs should be experimentally verified by molecular methods including sequencing, gene and protein array, RT-qPCR and western blot assays.

\section{Acknowledgements}

The present study was supported by Istanbul University Scientific Research Projects Department (grant no. 29278 and 40699). SEM Laboratory Company which is Agilent Technologies Distributor in Turkey supported the present study financially during the publication process. The authors would like to thank Professor Bulent Hayri ERMIS (Department of Obstetrics and Gynecology, Istanbul Medical Faculty, Istanbul, Turkey) who passed away on 24th of February 2016, for his considerable contribution to the current study. Additionally, many thanks to Mr Ismail Dolekcap for laboratory technical assistance and Dr Halil Ibrahim Kisakesen for bioinformatics assistance.

\section{References}

1. Wang Y, Lv Y, Wang L, Gong C, Sun J, Chen X, Chen Y, Yang L, Zhang Y, Yang X, et al: MicroRNAome in decidua: A new approach to assess the maintenance of pregnancy. Fertil Steril 103: 980-989 e6, 2015.

2. Zhu LJ, Chen YP, Chen BJ and Mei XH: Changes in reactive oxygen species, superoxide dismutase, and hypoxia-inducible factor- $1 \alpha$ levels in missed abortion. Int J Clin Exp Med 7: $2179-2184,2014$

3. Tang L, Gao C, Gao L, Cui Y and Liu J: Expression profile of micro-RNAs and functional annotation analysis of their targets in human chorionic villi from early recurrent miscarriage. Gene 576: 366-371, 2016.

4. Ventura W, Koide K, Hori K, Yotsumoto J, Sekizawa A, Saito H and Okai T: Placental expression of microRNA-17 and $-19 \mathrm{~b}$ is down-regulated in early pregnancy loss. Eur J Obstet Gynecol Reprod Biol 169: 28-32, 2013.

5. Larsen EC, Christiansen OB, Kolte AM and Macklon N: New insights into mechanisms behind miscarriage. BMC Med 11: $154,2013$.

6. Michels TC and Tiu AY: Second trimester pregnancy loss. Am Fam Physician 76: 1341-1346, 2007.

7. Dong F, Zhang Y, Xia F, Yang Y, Xiong S, Jin L and Zhang J: Genome-wide miRNA profiling of villus and decidua of recurrent spontaneous abortion patients. Reproduction 148: 33-41, 2014.

8. Rull K, Nagirnaja L and Laan M: Genetics of recurrent miscarriage: Challenges, current knowledge, future directions. Front Genet 3: 34, 2012.
9. Tsochandaridis M, Nasca L, Toga C and Levy-Mozziconacci A: Circulating MicroRNAs as clinical biomarkers in the predictions of pregnancy complications. Biomed Res Int 2015: 294954, 2015.

10. Esteller M: Non-coding RNAs in human disease. Nat Rev Genet 12: 861-874, 2011.

11. Luo L, Ye G, Nadeem L, Fu G, Yang BB, Honarparvar E, Dunk C, Lye S and Peng C: MicroRNA-378a-5p promotes trophoblast cell survival, migration and invasion by targeting Nodal. J Cell Sci 125: 3124-3132, 2012.

12. Li Y and Kowdley KV: MicroRNAs in common human diseases. Genomics Proteomics Bioinformatics 10: 246-253, 2012.

13. Li Z and Rana TM: Therapeutic targeting of microRNAs: Current status and future challenges. Nat Rev Drug Discov 13: 622-638, 2014

14. Gu Y, Sun J, Groome LJ and Wang Y: Differential miRNA expression profiles between the first and third trimester human placentas. Am J Physiol Endocrinol Metab 304: E836-E843, 2013.

15. Zhu XM, Han T, Sargent IL, Yin GW and Yao YQ: Differential expression profile of microRNAs in human placentas from preeclamptic pregnancies vs normal pregnancies. Am J Obstet Gynecol 200: 661.e1-7, 2009.

16. Fu G, Brkić J, Hayder H and Peng C: MicroRNAs in human placental development and pregnancy complications. Int J Mol Sci 14: 5519-5544, 2013.

17. Lo YM, Corbetta N, Chamberlain PF, Rai V, Sargent IL, Redman CW and Wainscoat JS: Presence of fetal DNA in maternal plasma and serum. Lancet 350: 485-487, 1997.

18. Zhao Z, Moley KH and Gronowski AM: Diagnostic potential for miRNAs as biomarkers for pregnancy-specific diseases. Clin Biochem 46: 953-960, 2013.

19. Lanza G, Ferracin M, Gafà R, Veronese A, Spizzo R, Pichiorri F, Liu CG, Calin GA, Croce CM and Negrini M: mRNA/microRNA gene expression profile in microsatellite unstable colorectal cancer. Mol Cancer 6: 54, 2007.

20. Livak KJ and Schmittgen TD: Analysis of relative gene expression data using real-time quantitative PCR and the 2(-Delta Delta C(T)) method. Methods 25: 402-408, 2001.

21. Siristatidis C, Vogiatzi P, Brachnis N, Liassidou A, Iliodromiti Z, Bettocchi S and Chrelias C: Review: MicroRNAs in assisted reproduction and their potential role in IVF failure. In Vivo 29: 169-175, 2015.

22. Sun YM, Lin KY and Chen YQ: Diverse functions of miR-125 family in different cell contexts. J Hematol Oncol 6: 6, 2013.

23. Hu Y, Liu CM, Qi L, He TZ, Shi-Guo L, Hao CJ, Cui Y, Zhang N, Xia HF and Ma X: Two common SNPs in pri-miR-125a alter the mature miRNA expression and associate with recurrent pregnancy loss in a Han-Chinese population. RNA Biol 85: 861-872, 2011.

24. Li D and Li J: Association of $\mathrm{miR}-34 \mathrm{a}-3 \mathrm{p} / 5 \mathrm{p}$, $\mathrm{miR}-141-3 \mathrm{p} / 5 \mathrm{p}$, and miR-24 in decidual natural killer cells with unexplained recurrent spontaneous abortion. Med Sci Monit 22: 922-929, 2016.

25. Salleh N and Giribabu N: Leukemia inhibitory factor: Roles in embryo implantation and in nonhormonal contraception. ScientificWorld Journal 2014: 201514, 2014.

26. Ninio-Many L, Grossman H, Shomron N, Chuderland D and Shalgi R: microRNA-125a-3p reduces cell proliferation and migration by targeting Fyn. J Cell Sci 126: 2867-2876, 2013.

27. Zhang H, Zhu X, Li N, Li D, Sha Z, Zheng X and Wang H: miR-125a-3p targets MTA1 to suppress NSCLC cell proliferation, migration, and invasion. Acta Biochim Biophys Sin (Shanghai) 47: 496-503, 2015.

28. Guo X, Wu Y and Hartley RS: MicroRNA-125a represses cell growth by targeting HuR in breast cancer. RNA Biol 6: 575-583, 2009.

29. Tiwari A, Shivananda S, Gopinath KS and Kumar A: MicroRNA-125a reduces proliferation and invasion of oral squamous cell carcinoma cells by targeting estrogen-related receptor $\alpha$ : Implications for cancer therapeutics. J Biol Chem 289: 32276-32290, 2014.

30. Choi HK, Choi BC, Lee SH, Kim JW, Cha KY and Baek KH: Expression of angiogenesis- and apoptosis-related genes in chorionic villi derived from recurrent pregnancy loss patients. Mol Reprod Dev 66: 24-31, 2003.

31. Gu H, Li H, Zhang L, Luan H, Huang T, Wang L, Fan Y, Zhang Y, Liu X, Wang W and Yuan Z: Diagnostic role of microRNA expression profile in the serum of pregnant women with fetuses with neural tube defects. J Neurochem 122: 641-649, 2012. 
32. Nardelli C, Iaffaldano L, Ferrigno M, Labruna G, Maruotti GM, Quaglia F, Capobianco V, Di Noto R, Del Vecchio L, Martinelli $\mathrm{P}$, et al: Characterization and predicted role of the microRNA expression profile in amnion from obese pregnant women. Int J Obes (Lond) 38: 466-469, 2014.

33. Fisher JN, Terao M, Fratelli M, Kurosaki M, Paroni G, Zanetti A, Gianni M, Bolis M, Lupi M, Tsykin A, et al: MicroRNA networks regulated by all-trans retinoic acid and Lapatinib control the growth, survival and motility of breast cancer cells. Oncotarget 6: 13176-13200, 2015.

34. Sand M, Skrygan M, Sand D, Georgas D, Gambichler T, Hahn SA, Altmeyer P and Bechara FG: Comparative microarray analysis of microRNA expression profiles in primary cutaneous malignant melanoma, cutaneous malignant melanoma metastases, and benign melanocytic nevi. Cell Tissue Res 351: 85-98, 2013.

35. Peng J, Feng Y, Rinaldi G, Levine P, Easley S, Martinez E, Hashmi S, Sadeghi N, Brindley PJ, Mulvenna JP, et al: Profiling miRNAs in nasopharyngeal carcinoma FFPE tissue by microarray and next generation sequencing. Genom Data 2: 285-289, 2014.

36. Chiyomaru T, Yamamura S, Fukuhara S, Hidaka H, Majid S, Saini S, Arora S, Deng G, Shahryari V, Chang I, et al: Genistein up-regulates tumor suppressor microRNA-574-3p in prostate cancer. PLoS One 8: e58929, 2013.

37. Gitenay D, Lallet-Daher H and Bernard D: Caspase-2 regulates oncogene-induced senescence. Oncotarget 5: 5845-5847, 2014.

38. Vanbekbergen N, Hendrickx M and Leyns L: Growth differentiation Factor 11 is an encephalic regionalizing factor in neural differentiated mouse embryonic stem cells. BMC Res Notes 7 : 766, 2014.

39. Yang L, Wang L and Zheng Y: Gene targeting of Cdc42 and Cdc42GAP affirms the critical involvement of Cdc42 in filopodia induction, directed migration, and proliferation in primary mouse embryonic fibroblasts. Mol Biol Cell 17: 4675-4685, 2006.

40. Large MJ, Wetendorf M, Lanz RB, Hartig SM, Creighton CJ Mancini MA, Kovanci E, Lee KF, Threadgill DW, Lydon JP, et al: The epidermal growth factor receptor critically regulates endometrial function during early pregnancy. PLoS Genet 10 : e1004451, 2014

41. Bao SH, Shuai W, Tong J, Wang L, Chen P and Duan T: Increased Dickkopf-1 expression in patients with unexplained recurrent spontaneous miscarriage. Clin Exp Immunol 172: 437-443, 2013

42. Jin YX, Cui XS, Han YJ and Kim NH: Leptin accelerates pronuclear formation following intracy toplasmic sperm injection of porcine oocytes: Possible role for MAP kinase inactivation. Anim Reprod Sci 115: 137-148, 2009.

43. Xiao B, Xue X, Hu F, Sun R, Chen Q, Yang M and Zhang W: Expression and regulatory mechanism of microRNA-155 in the villi of patients with unexplained recurrent spontaneous abortion patients. Zhonghua Fu Chan Ke Za Zhi 49: 130-134, 2014 (In Chinese)

44. Qin W, Tang Y, Yang N, Wei X and Wu J: Potential role of circulating microRNAs as a biomarker for unexplained recurrent spontaneous abortion. Fertil Steril 105: 1247-1254 e3, 2016

45. Luque A, Farwati A, Crovetto F, Crispi F, Figueras F, Gratacós E and Aran JM: Usefulness of circulating microRNAs for the prediction of early preeclampsia at first-trimester of pregnancy. Sci Rep 4: 4882, 2014

46. Al-Shorafa $\mathrm{H}$ and Sharif FA: MicroRNA in a case of unexplained recurrent pregnancy loss. J Clin Case Rep 02: 238, 2012.
47. Petracco R, Grechukhina O, Popkhadze S, Massasa E, Zhou Y and Taylor HS: MicroRNA 135 regulates HOXA10 expression in endometriosis. J Clin Endocrinol Metab 96: E1925-E1933, 2011.

48. Shi H, Ji Y, Zhang D, Liu Y and Fang P: MiR-135a inhibits migration and invasion and regulates EMT-related marker genes by targeting KLF8 in lung cancer cells. Biochem Biophys Res Commun 465: 125-130, 2015

49. Navarro A, Diaz T, Martinez A, Gaya A, Pons A, Gel B, Codony C, Ferrer G, Martinez C, Montserrat E and Monzo M: Regulation of JAK2 by miR-135a: Prognostic impact in classic Hodgkin lymphoma. Blood 114: 2945-2951, 2009.

50. Ishihara N, Matsuo H, Murakoshi H, Laoag-Fernandez JB, Samoto T and Maruo T: Increased apoptosis in the syncytiotrophoblast in human term placentas complicated by either preeclampsia or intrauterine growth retardation. Am J Obstet Gynecol 185: 158-166, 2002.

51. Zhang ZW,LiH, Chen SS, Li Y, Cui ZY and Ma J: MicroRNA-122 regulates caspase- 8 and promotes the apoptosis of mouse cardiomyocytes. Braz J Med Biol Res 50: e5760, 2017.

52. Ma L, Liu J, Shen J, Liu L, Wu J, Li W, Luo J, Chen Q and Qian C: Expression of miR-122 mediated by adenoviral vector induces apoptosis and cell cycle arrest of cancer cells. Cancer Biol Ther 9: 554-561, 2010.

53. Tsai WC, Hsu PW, Lai TC, Chau GY, Lin CW, Chen CM, Lin CD, Liao YL, Wang JL, Chau YP, et al: MicroRNA-122, a tumor suppressor microRNA that regulates intrahepatic metastasis of hepatocellular carcinoma. Hepatology 49: 1571-1582, 2009.

54. LasabováZ, Vazan M,Zibolenova J and Svecova I: Overexpression of miR-21 and miR-122 in preeclamptic placentas. Neuro Endocrinol Lett 36: 695-699, 2015.

55. Ding Z, Wang X, Schnackenberg L, Khaidakov M, Liu S, Singla S, Dai Y and Mehta JL: Regulation of autophagy and apoptosis in response to ox-LDL in vascular smooth muscle cells, and the modulatory effects of the microRNA hsa-let-7g. Int J Cardiol 168: 1378-1385, 2013.

56. Chen Z, Lai TC, Jan YH, Lin FM, Wang WC, Xiao H, Wang YT, Sun W, Cui X, Li YS, et al: Hypoxia-responsive miRNAs target argonaute 1 to promote angiogenesis. J Clin Invest 123: 1057-1067, 2013.

57. Du H, Sarno J and Taylor HS: HOXA10 inhibits Kruppel-like factor 9 expression in the human endometrial epithelium. Biol Reprod 83: 205-211, 2010.

58. Tokyol C, Aktepe F, Husniye Dilek F and Yilmazer M: Comparison of placental PTEN and beta1 integrin expression in early spontaneous abortion, early and late normal pregnancy. Ups J Med Sci 113: 235-242, 2008.

59. Bao MH, Feng X, Zhang YW, Lou XY, Cheng Y and Zhou HH: Let-7 in cardiovascular diseases, heart development and cardiovascular differentiation from stem cells. Int J Mol Sci 14: 23086-23102, 2013

60. Qin B, Xiao B, Liang D, Li Y, Jiang T and Yang H: MicroRNA let-7c inhibits Bcl-xl expression and regulates ox-LDL-induced endothelial apoptosis. BMB Rep 45: 464-469, 2012.

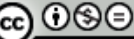

This work is licensed under a Creative Commons Attribution-NonCommercial-NoDerivatives 4.0 International (CC BY-NC-ND 4.0) License. 